\title{
Integral mean estimates for polynomials whose zeros are within a circle
}

\author{
Gulshan Singh ${ }^{1 *}$ and WM Shah ${ }^{2}$
}

\author{
* Correspondence: \\ gulshansingh1@rediffmail.com \\ 'Bharathiar University, Coimbatore, \\ TN, 641046, India \\ Full list of author information is \\ available at the end of the article
}

\section{Abstract}

Let $P(z)$ be a polynomial of degree $n$ having all its zeros in $|z| \leq K \leq 1$, then for each $\delta>0, p>1, q>1$ with $\frac{1}{p}+\frac{1}{q}=1$, Aziz and Ahmad (Glas Mat Ser III 31:229-237, 1996) proved that

$$
n\left\{\int_{0}^{2 \pi}\left|P\left(e^{i \theta}\right)\right|^{\delta} d \theta\right\}^{\frac{1}{\delta}} \leq\left\{\int_{0}^{2 \pi}\left|1+K e^{i \theta}\right|^{q \delta} d \theta\right\}^{\frac{1}{q \delta}}\left\{\int_{0}^{2 \pi}\left|P^{\prime}\left(e^{i \theta}\right)\right|^{p \delta} d \theta\right\}^{\frac{1}{p \delta}} .
$$

In this paper, we extend the above inequality to the class of polynomials $P(z):=a_{n} z^{n}+\sum_{j=\mu}^{n} a_{n-j} z^{n-j}, 1 \leq \mu \leq n$, having all its zeros in $|z| \leq K \leq 1$, and obtain a generalization as well as refinement of the above result.

\section{Mathematics Subject Classification (2000)}

30A 10, 30C10, 30C 15

Keywords: Derivative of a polynomial, Integral mean estimates, Inequalities in complex domain

\section{Introduction and statement of results}

Let $P(z)$ be a polynomial of degree $\mathrm{n}$ and $P^{\prime}(z)$ be its derivative. If $P(z)$ has all its zeros in $|z| \leq 1$, then it was shown by Turan [1] that

$$
\operatorname{Max}_{|z|=1}\left|P^{\prime}(z)\right| \geq \frac{n}{2} \operatorname{Max}_{|z|=1}|P(z)|
$$

Inequality (1) is best possible with equality for $P(z)=\alpha z^{n}+\beta$, where $|\alpha|=|\beta|$. As an extension of (1), Malik [2] proved that if $P(z)$ has all its zeros in $|z| \leq K$, where $K \leq 1$, then

$$
\operatorname{Max}_{|z|=1}\left|P^{\prime}(z)\right| \geq \frac{n}{1+K} \operatorname{Max}_{|z|=1}|P(z)| .
$$

Malik [3] also obtained a generalization of (1) in the sense that the right-hand side of (1) is replaced by a factor involving the integral mean of $|P(z)|$ on $|z|=1$. In fact, he proved the following: 
Theorem A. If $P(z)$ has all its zeros in $|z| \leq 1$, then for each $\delta>0$

$$
n\left\{\int_{0}^{2 \pi}\left|P\left(e^{i \theta}\right)\right|^{\delta} d \theta\right\}^{\frac{1}{\delta}} \leq\left\{\int_{0}^{2 \pi}\left|1+e^{i \theta}\right|^{\delta} d \theta\right\}^{\frac{1}{\delta}} \operatorname{Max}_{|z|=1}\left|P^{\prime}(z)\right| .
$$

The result is sharp, and equality in (3) holds for $P(z)=(z+1)^{n}$. If we let $\delta \rightarrow \infty$ in (3), we get (1).

As a generalization of Theorem A, Aziz and Shah [4] proved the following:

Theorem B. If $P(z):=a_{n} z^{n}+\sum_{j=\mu}^{n} a_{n-j} z^{n-j}, 1 \leq \mu \leq n$ is a polynomial of degree $n$ having all its zeros in the disk $|z| \leq K, K \leq 1$, then for each $\delta>0$,

$$
n\left\{\int_{0}^{2 \pi}\left|P\left(e^{i \theta}\right)\right|^{\delta} d \theta\right\}^{\frac{1}{\delta}} \leq\left\{\int_{0}^{2 \pi}\left|1+K^{\mu} e^{i \theta}\right|^{\delta} d \theta\right\}^{\frac{1}{\delta}} \operatorname{Max}|z|=1\left|P^{\prime}(z)\right| .
$$

Aziz and Ahmad [5] generalized (3) in the sense that $\operatorname{Max}_{|z|=1}\left|P^{\prime}(z)\right|$ on $|z|=1$ on the right-hand side of (3) is replaced by a factor involving the integral mean of $\left|P^{\prime}(z)\right|$ on $|z|=1$ and proved the following:

Theorem C. If $P(z)$ is a polynomial of degree $n$ having all its zeros in $|z| \leq K \leq 1$, then for $\delta>0, p>1, q>1$ with $\frac{1}{p}+\frac{1}{q}=1$,

$$
n\left\{\int_{0}^{2 \pi}\left|P\left(e^{i \theta}\right)\right|^{\delta} d \theta\right\}^{\frac{1}{\delta}} \leq\left\{\int_{0}^{2 \pi}\left|1+K e^{i \theta}\right|^{q \delta} d \theta\right\}^{\frac{1}{q \delta}}\left\{\int_{0}^{2 \pi}\left|P^{\prime}\left(e^{i \theta}\right)\right|^{p \delta} d \theta\right\}^{\frac{1}{p \delta}} .
$$

If we let $p \rightarrow \infty$ (so that $q \rightarrow 1$ ) in (5), we get (3).

In this paper, we consider a class of polynomials $P(z):=a_{n} z^{n}+\sum_{j=\mu}^{n} a_{n-j} z^{n-j}, 1 \leq \mu \leq$ $n$, having all the zeros in $|z| \leq K \leq 1$, and thereby obtain a more general result by proving the following:

Theorem 1. If $P(z):=a_{n} z^{n}+\sum_{j=\mu}^{n} a_{n-j} z^{n-j}, 1 \leq \mu \leq n$ is a polynomial of degree $n$ having all its zeros in the disk $|z| \leq K, K \leq 1$, then for each $\delta>0, q>1, p>1$ with $\frac{1}{p}+\frac{1}{q}=1$ and for every complex number $\lambda$ with $|\lambda|<1$

$$
\begin{aligned}
& n\left\{\int_{0}^{2 \pi}\left|P\left(e^{i \theta}\right)+\lambda m\right|^{\delta} d \theta\right\}^{\frac{1}{\delta}} \\
& \leq\left\{\int_{0}^{2 \pi}\left|1+\left[\frac{n\left|a_{n}\right| K^{2 \mu}+\mu\left|a_{n-\mu}\right| K^{\mu-1}}{n\left|a_{n}\right| K^{\mu-1}+\mu\left|a_{n-\mu}\right|}\right] e^{i \theta}\right|^{q \delta} d \theta\right\}^{\frac{1}{q \delta}}\left\{\int_{0}^{2 \pi}\left|P^{\prime}\left(e^{i \theta}\right)\right|^{p \delta} d \theta\right\}^{\frac{1}{p \delta}},
\end{aligned}
$$

where $m=\operatorname{Min}_{|z|=K}|P(z)|$.

If we take $\lambda=0$ in Theorem 1, we get the following:

Corollary 1. If $P(z):=a_{n} z^{n}+\sum_{j=\mu}^{n} a_{n-j} z^{n-j}, 1 \leq \mu \leq n$ is a polynomial of degree $n$ having all its zeros in the disk $|z| \leq K, K \leq 1$, then for each $\delta>0, q>1, p>1$ with 


$$
\begin{aligned}
& n\left\{\int_{0}^{2 \pi}\left|P\left(e^{i \theta}\right)\right|^{\delta} d \theta\right\}^{\frac{1}{\delta}} \\
& \leq\left\{\left.\int_{0}^{2 \pi}\left|1+\left[\frac{n\left|a_{n}\right| K^{2 \mu}+\mu\left|a_{n-\mu}\right| K^{\mu-1}}{n\left|a_{n}\right| K^{\mu-1}+\mu\left|a_{n-\mu}\right|}\right] e^{i \theta}\right|\right|^{\phi \delta} d \theta\right\}^{\frac{1}{\phi \delta}}\left\{\int_{0}^{2 \pi}\left|P^{\prime}\left(e^{i \theta}\right)\right|^{p \delta} d \theta\right\}^{\frac{1}{p \delta}}, \\
& n\left\{\int_{0}^{2 \pi}\left|P\left(e^{i \theta}\right)\right|^{\delta} d \theta\right\}^{\frac{1}{\delta}} \\
& \leq\left\{\int_{0}^{2 \pi}\left|1+\left[\frac{n\left|a_{n}\right| K^{2 \mu}+\mu\left|a_{n-\mu}\right| K^{\mu-1}}{n\left|a_{n}\right| K^{\mu-1}+\mu\left|a_{n-\mu}\right|}\right] e^{i \theta}\right|^{q \delta} d \theta\right\}^{\frac{1}{q \delta}}\left\{\int_{0}^{2 \pi}\left|P^{\prime}\left(e^{i \theta}\right)\right|^{p \delta} d \theta\right\}^{\frac{1}{p \delta}},
\end{aligned}
$$

For $\mu=1$ in Theorem 1, we have the following:

Corollary 2. If $P(z):=\sum_{j=0}^{n} a_{j} z^{i}$ is a polynomial of degree $n$ having all its zeros in the disk $|z| \leq K, K \leq 1$, then for each $\delta>0, q>1, p>1$ with $\frac{1}{p}+\frac{1}{q}=1$,

$$
\begin{aligned}
& n\left\{\int_{0}^{2 \pi}\left|P\left(e^{i \theta}\right)+\lambda m\right|^{\delta} d \theta\right\}^{\frac{1}{\delta}} \\
& \quad \leq\left\{\int_{0}^{2 \pi}\left|1+\left[\frac{n\left|a_{n}\right| K^{2}+\left|a_{n-1}\right|}{n\left|a_{n}\right|+\left|a_{n-1}\right|}\right] e^{i \theta}\right|^{q \delta} d \theta\right\}^{\frac{1}{q \delta}}\left\{\int_{0}^{2 \pi}\left|P^{\prime}\left(e^{i \theta}\right)\right|^{p \delta} d \theta\right\}^{\frac{1}{p \delta}},
\end{aligned}
$$

where $m=\operatorname{Min}_{|z|=K}|P(z)|$.

Remark 1: Since all the zeros of $P(z)$ lie in $|z| \leq K$, therefore, $\frac{1}{n}\left|\frac{a_{n-1}}{a_{n}}\right| \leq K K \leq 1$, it can be easily verified that

$$
\frac{n\left|a_{n}\right| K^{2}+\left|a_{n-1}\right|}{n\left|a_{n}\right|+\left|a_{n-1}\right|} \leq K
$$

It shows that for $\lambda=0$, Corollary 2 provides a refinement of the result of Aziz and Ahmad [5].

The next result immediately follows from Theorem 1 , if we let $p \rightarrow \infty$ (so that $q \rightarrow$ 1)

Corollary 3. If $P(z):=a_{n} z^{n}+\sum_{j=\mu}^{n} a_{n-j} z^{n-j}, 1 \leq \mu \leq n$ is a polynomial of degree $n$ having all its zeros in the disk $|z| \leq K, K \leq 1$, then for each $\delta>0$ and for every complex number $\lambda$ with $|\lambda|<1$

$$
\begin{aligned}
& n\left\{\int_{0}^{2 \pi}\left|P\left(e^{i \theta}\right)+\lambda m\right|^{\delta} d \theta\right\}^{\frac{1}{\delta}} \\
& \quad \leq\left\{\int_{0}^{2 \pi}\left|1+\left[\frac{n\left|a_{n}\right| K^{2 \mu}+\mu\left|a_{n-\mu}\right| K^{\mu-1}}{n\left|a_{n}\right| K^{\mu-1}+\mu\left|a_{n-\mu}\right|}\right] e^{i \theta}\right|^{\delta} d \theta\right\}^{\frac{1}{\delta}} \operatorname{Max}_{|z|=1}\left|P^{\prime}(z)\right| .
\end{aligned}
$$


Also if we let $\delta \rightarrow \infty$ in the Corollary 3 and note that

$$
\lim _{\delta \rightarrow \infty}\left\{\frac{1}{2 \pi} \int_{0}^{2 \pi}\left|P\left(e^{i \theta}\right)\right|^{\delta} \mathrm{d} \theta\right\}^{\frac{1}{\delta}}=\operatorname{Max}_{|z|=1}|P(z)|,
$$

we get from (9)

$$
n \operatorname{Max}_{|z|=\mid}|P(z)+\lambda m| \leq \frac{n\left|a_{n}\right|\left(K^{2 \mu}+K^{\mu-1}\right)+\mu\left|a_{n-\mu}\right|\left(1+K^{\mu-1}\right)}{n\left|a_{n}\right| K^{\mu-1}+\mu\left|a_{n-\mu}\right|} \operatorname{Max}_{|z|=1}\left|P^{\prime}(z)\right| \text { for }|z|=1 .
$$

If $z_{0}$ be such that $\operatorname{Max}_{|z|=1}|P(z)|=\left|P\left(z_{0}\right)\right|$, then from (10), we have

$$
\begin{aligned}
& n\left|P\left(z_{0}\right)+\lambda m\right| \leq \\
& \frac{n\left|a_{n}\right|\left(K^{2 \mu}+K^{\mu-1}\right)+\mu\left|a_{n-\mu}\right|\left(1+K^{\mu-1}\right)}{n\left|a_{n}\right| K^{\mu-1}+\mu\left|a_{n-\mu}\right|} M a x_{|z|=1}\left|P^{\prime}(z)\right| \quad \text { for } \quad|z|=1 .
\end{aligned}
$$

Choosing an argument of $\lambda$ such that

$$
\left|P\left(z_{0}\right)+\lambda m\right|=\left|P\left(z_{0}\right)\right|+|\lambda| m,
$$

we get

$$
n\left(\left|P\left(z_{0}\right)\right|+|\lambda| m\right) \leq \frac{n\left|a_{n}\right|\left(K^{2 \mu}+K^{\mu-1}\right)+\mu\left|a_{n-\mu}\right|\left(1+K^{\mu-1}\right)}{n\left|a_{n}\right| K^{\mu-1}+\mu\left|a_{n-\mu}\right|} \operatorname{Max}_{|z|=1}\left|P^{\prime}(z)\right| .
$$

From inequality (11), we conclude the following:

Corollary 4. If $P(z):=a_{n} z^{n}+\sum_{j=\mu}^{n} a_{n-j} z^{n-j}, 1 \leq \mu \leq n$ is a polynomial of degree $n$ having all its zeros in the disk $|z| \leq K, K \leq 1$, then for $0 \leq t \leq 1$, we have

$$
\begin{gathered}
\operatorname{Max}_{|z|=1}\left|P^{\prime}(z)\right| \geq \\
n \frac{n\left|a_{n}\right| K^{\mu-1}+\mu\left|a_{n-\mu}\right|}{n\left|a_{n}\right|\left(K^{2 \mu}+K^{\mu-1}\right)+\mu\left|a_{n-\mu}\right|\left(1+K^{\mu-1}\right)}\left\{M a z_{|z|=1}|P(z)|+t \operatorname{Min}_{|z|=K}|P(z)|\right\} .
\end{gathered}
$$

Further, if we take $K=t=\mu=1$ in the Corollary 4, we get a result of Aziz and Dawood [6].

\section{Lemmas}

For the proof of this theorem, we need the following lemmas.

The first lemma is due to Qazi [7].

Lemma 1. If $P(z):=a_{0}+\sum_{j=\mu}^{n} a_{j} z^{j}, 1 \leq \mu \leq n$ is a polynomial of degree $n$ having no zeros in the disk $|z|<K, K \geq 1$, then

$$
\left[\frac{\left.n\left|a_{0}\right|\right|^{\mu+1}+\mu\left|a_{\mu}\right| K^{2 \mu}}{n\left|a_{0}\right|+\mu\left|a_{\mu}\right| K^{\mu+1}}\right]\left|P^{\prime}(z)\right| \leq\left|Q^{\prime}(z)\right| \text { for }|z|=1,
$$

whereQ $(z)=z^{n} \overline{P\left(\frac{1}{\bar{z}}\right)}$ and $\frac{\mu}{n}\left|\frac{a_{\mu}}{a_{0}}\right| K^{\mu} \leq 1$.

Lemma 2. If $P(z):=a_{n} z^{n}+\sum_{j=\mu}^{n} a_{n-j} z^{n-j}$ is a polynomial of degree $n$ having all its zeros in the disk $|z| \leq K \leq 1$, then

$$
\left|Q^{\prime}(z)\right| \leq\left[\frac{n\left|a_{n}\right| K^{2 \mu}+\mu\left|a_{n-\mu}\right| K^{\mu-1}}{n\left|a_{n}\right| K^{\mu-1}+\mu\left|a_{n-\mu}\right|}\right]\left|P^{\prime}(z)\right| \quad \text { for }|z|=1,1 \leq \mu \leq n,
$$

where $Q(z)=z^{n} \overline{P\left(\frac{1}{\bar{z}}\right)}$. 


\section{Proof of Lemma 2}

Since all the zeros of $P(z)$ lie in $|z| \leq K \leq 1$, therefore all the zeros of $Q(z)=z^{n} \overline{P\left(\frac{1}{\bar{z}}\right)}$ lie in $|z| \geq \frac{1}{K} \geq 1$. Hence, applying Lemma 1 to the polynomial $Q(z):=\bar{a}_{n}+\sum_{j=\mu}^{n} \bar{a}_{n-j} z^{j}$, we get

$$
\left[\frac{n\left|a_{n}\right| \frac{1}{K^{\mu+1}}+\mu\left|a_{n-\mu}\right| \frac{1}{K^{2 \mu}}}{n\left|a_{n}\right|+\mu\left|a_{n-\mu}\right| \frac{1}{K^{\mu+1}}}\right]\left|Q^{\prime}(z)\right| \leq\left|P^{\prime}(z)\right| .
$$

Or, equivalently

$$
\left|Q^{\prime}(z)\right| \leq\left[\frac{n\left|a_{n}\right| K^{2 \mu}+\mu\left|a_{n-\mu}\right| K^{\mu-1}}{n\left|a_{n}\right| K^{\mu-1}+\mu\left|a_{n-\mu}\right|}\right]\left|P^{\prime}(z)\right|, \quad|z|=1 .
$$

This proves Lemma 2.

Remark 1: Lemma 3 of Govil and Mc Tume [8] is a special case of this lemma when $\mu=1$.

\section{Proof of Theorem 1}

Let $Q(z)=z^{n} \overline{P\left(\frac{1}{\bar{z}}\right)} z$, we have $P(z)=z^{n} \overline{Q\left(\frac{1}{\bar{z}}\right)}$. This gives

$$
P^{\prime}(z)=n z^{n-1} \overline{Q\left(\frac{1}{\bar{z}}\right)}-z^{n-2} \overline{Q^{\prime}\left(\frac{1}{\bar{z}}\right)} .
$$

Equivalently,

$$
z P^{\prime}(z)=n z^{n} \overline{Q\left(\frac{1}{\bar{z}}\right)}-z^{n-1} \overline{Q^{\prime}\left(\frac{1}{\bar{z}}\right)} .
$$

This implies

$$
\left|P^{\prime}(z)\right|=\left|n Q(z)-z Q^{\prime}(z)\right| \quad \text { for } \quad|z|=1 .
$$

Let $m=\operatorname{Min}_{|z|=K}|P(z)|$, so that $m \leq|P(z)|$ for $|z|=K$. Therefore, for every complex number $\lambda$ with $|\lambda|<1$, we have $|m \lambda|<|P(z)|$ on $|z|=K$. Since $P(z)$ has all its zeros in $|z| \leq K \leq 1$, by Rouche's theorem, it follows that all the zeros of the polynomial $G(z)=$ $P(z)+\lambda m$ lie in $|z| \leq K \leq 1$.

If $H(z)=z^{n} \overline{G\left(\frac{1}{\bar{z}}\right)}=Q(z)+m \bar{\lambda} z^{n}$, then by applying Lemma 2 to the polynomial $G(z)$ $=P(z)+\lambda m$, we have for $|z|=1$

$$
\left|H^{\prime}(z)\right| \leq\left[\frac{n\left|a_{n}\right| K^{2 \mu}+\mu\left|a_{n-\mu}\right| K^{\mu-1}}{n\left|a_{n}\right| K^{\mu-1}+\mu\left|a_{n-\mu}\right|}\right]\left|G^{\prime}(z)\right|, \quad 1 \leq \mu \leq n .
$$

This gives

$$
\left|Q^{\prime}(z)+n m \bar{\lambda} z^{n-1}\right| \leq\left[\frac{n\left|a_{n}\right| K^{2 \mu}+\mu\left|a_{n-\mu}\right| K^{\mu-1}}{n\left|a_{n}\right| K^{\mu-1}+\mu\left|a_{n-\mu}\right|}\right]\left|P^{\prime}(z)\right|, \quad 1 \leq \mu \leq n .
$$

Using (14) in (15), we get

$$
\begin{aligned}
& \left|Q^{\prime}(z)+n m \bar{\lambda} z^{n-1}\right| \leq\left[\frac{n\left|a_{n}\right| K^{2 \mu}+\mu\left|a_{n-\mu}\right| K^{\mu-1}}{n\left|a_{n}\right| K^{\mu-1}+\mu\left|a_{n-\mu}\right|}\right]\left|n Q(z)-z Q^{\prime}(z)\right| \text { for }|z|= \\
& 1 \leq \mu \leq n .
\end{aligned}
$$


Since $P(z)$ has all its zeros in $|z| \leq K \leq 1$, by Gauss\{Lucas theorem so does $P^{\prime}(z)$. It follows that $n Q(z)-z Q^{\prime}(z)$, which is simply (see (12))

$$
z^{n-1} \overline{P^{\prime}\left(\frac{1}{\bar{z}}\right)}
$$

has all its zeros in $|z| \geq \frac{1}{K} \geq 1$. Hence,

$$
W(z)=\left[\frac{n\left|a_{n}\right| K^{\mu-1}+\mu\left|a_{n-\mu}\right|}{n\left|a_{n}\right| K^{2 \mu}+\mu\left|a_{n-\mu}\right| K^{\mu-1}}\right] \cdot \frac{z\left(Q^{\prime}(z)+n m \bar{\lambda} z^{n-1}\right)}{\left(n Q(z)-z Q^{\prime}(z)\right)}
$$

is analytic for $|z|<1,|W(z)| \leq 1$ for $|z|=1$ and $W(0)=0$. Thus, the function

$$
1+\left[\frac{n\left|a_{n}\right| K^{2 \mu}+\mu\left|a_{n-\mu}\right| K^{\mu-1}}{n\left|a_{n}\right| K^{\mu-1}+\mu\left|a_{n-\mu}\right|}\right] \cdot W(z)
$$

is subordinate to the function

$$
1+\left[\frac{n\left|a_{n}\right| K^{2 \mu}+\mu\left|a_{n-\mu}\right| K^{\mu-1}}{n\left|a_{n}\right| K^{\mu-1}+\mu\left|a_{n-\mu}\right|}\right] z
$$

for $|z|<1$. Hence, by a property of subordination (for reference see [[9], p. 36, Theorem 1.6.17] or [[10], p. 454] or [11]), we have for each $\delta>0$ and $0 \leq \theta<2 \pi$,

$$
\begin{gathered}
\int_{0}^{2 \pi}\left|1+\left[\frac{n\left|a_{n}\right| K^{2 \mu}+\mu\left|a_{n-\mu}\right| K^{\mu-1}}{n\left|a_{n}\right| K^{\mu-1}+\mu\left|a_{n-\mu}\right|}\right] W\left(e^{i \theta}\right)\right|^{\delta} \mathrm{d} \theta \\
\leq \int_{0}^{2 \pi}\left|1+\left[\frac{n\left|a_{n}\right| K^{2 \mu}+\mu\left|a_{n-\mu}\right| K^{\mu-1}}{n\left|a_{n}\right| K^{\mu-1}+\mu\left|a_{n-\mu}\right|}\right] \mathrm{e}^{i \theta}\right|^{\delta} \mathrm{d} \theta .
\end{gathered}
$$

Also from (17), we have

$$
1+\left[\frac{n\left|a_{n}\right| K^{2 \mu}+\mu\left|a_{n-\mu}\right| K^{\mu-1}}{n\left|a_{n}\right| K^{\mu-1}+\mu\left|a_{n-\mu}\right|}\right] W(z)=\frac{n\left(Q(z)+m \bar{\lambda} z^{n}\right)}{n Q(z)-z Q^{\prime}(z)} .
$$

Therefore,

$$
n\left|Q(z)+m \bar{\lambda} z^{n}\right|=\left|1+\left[\frac{n\left|a_{n}\right| K^{2 \mu}+\mu\left|a_{n-\mu}\right| K^{\mu-1}}{n\left|a_{n}\right| K^{\mu-1}+\mu\left|a_{n-\mu}\right|}\right] W(z)\right|\left|n Q(z)-z Q^{\prime}(z)\right|,
$$

which implies

$$
n|H(z)|=\left|1+\left[\frac{n\left|a_{n}\right| K^{2 \mu}+\mu\left|a_{n-\mu}\right| K^{\mu-1}}{n\left|a_{n}\right| K^{\mu-1}+\mu\left|a_{n-\mu}\right|}\right] W(z)\right|\left|n Q(z)-z Q^{\prime}(z)\right| .
$$

Using (14) and the fact that $|H(z)|=|G(z)|=|P(z)+\lambda m|$ for $|z|=1$, we get from (20)

$$
n|P(z)+\lambda m|=\left|1+\left[\frac{n\left|a_{n}\right| K^{2 \mu}+\mu\left|a_{n-\mu}\right| K^{\mu-1}}{n\left|a_{n}\right| K^{\mu-1}+\mu\left|a_{n-\mu}\right|}\right] W(z)\right|\left|P^{\prime}(z)\right| \quad \text { for } \quad|z|=1 .
$$


Hence, for each $\delta>0$ and $0 \leq \theta<2 \pi$, we have

$$
\begin{aligned}
& n^{\delta} \int_{0}^{2 \pi}\left|P\left(e^{i \theta}\right)+\lambda m\right|^{\delta} \mathrm{d} \theta \\
& \quad=\int_{0}^{2 \pi}\left|1+\left[\frac{n\left|a_{n}\right| K^{2 \mu}+\mu\left|a_{n-\mu}\right| K^{\mu-1}}{n\left|a_{n}\right| K^{\mu-1}+\mu\left|a_{n-v}\right|}\right] W\left(e^{i \theta}\right)\right|^{\delta}\left|P^{\prime}\left(e^{i \theta}\right)\right|^{\delta} \mathrm{d} \theta .
\end{aligned}
$$

This gives with the help of Hölder's inequality for $p>1, q>1$, with $\frac{1}{p}+\frac{1}{q}=1$ and for every $\delta>0$,

$$
\begin{aligned}
& n^{\delta} \int_{0}^{2 \pi}\left|P\left(e^{i \theta}\right)+\lambda m\right|^{\delta} \mathrm{d} \theta \\
& \quad \leq\left\{\int_{0}^{2 \pi}\left|1+\left[\frac{n\left|a_{n}\right| K^{2 \mu}+\mu\left|a_{n-\mu}\right| K^{\mu-1}}{n\left|a_{n}\right| K^{\mu-1}+\mu\left|a_{n-\mu}\right|}\right] W\left(\mathrm{e}^{i \theta}\right)\right|^{q \delta} \mathrm{d} \theta\right\}^{\frac{1}{q}}\left\{\int_{0}^{2 \pi}\left|P^{\prime}\left(\mathrm{e}^{i \theta}\right)\right|^{p \delta} \mathrm{d} \theta\right\}^{\frac{1}{p}}
\end{aligned}
$$

Combining (18) and (22), we get for $\delta>0$ and $0 \leq \theta<2 \pi$,

$$
\begin{aligned}
& n^{\delta} \int_{0}^{2 \pi}\left|P\left(\mathrm{e}^{i \theta}\right)+\lambda m\right|^{\delta} \mathrm{d} \theta \\
& \quad \leq\left\{\int_{0}^{2 \pi}\left|1+\left[\frac{n\left|a_{n}\right| K^{2 \mu}+\mu\left|a_{n-\mu}\right| K^{\mu-1}}{n\left|a_{n}\right| K^{\mu-1}+\mu\left|a_{n-\mu}\right|}\right] \mathrm{e}^{i \theta}\right|^{q \delta} \mathrm{d} \theta\right\}^{\frac{1}{q}}\left\{\int_{0}^{2 \pi}\left|P^{\prime}\left(\mathrm{e}^{i \theta}\right)\right|^{\phi \delta} \mathrm{d} \theta\right\}^{\frac{1}{p}}
\end{aligned}
$$

This is equivalent to

$$
\begin{aligned}
& n\left\{\int_{0}^{2 \pi}\left|P\left(\mathrm{e}^{i \theta}\right)+\lambda m\right|^{\delta} \mathrm{d} \theta\right\}^{\frac{1}{\delta}} \\
& \quad \leq\left\{\left.\int_{0}^{2 \pi}\left|1+\left[\frac{n\left|a_{n}\right| K^{2 \mu}+\mu\left|a_{n-\mu}\right| K^{\mu-1}}{n\left|a_{n}\right| K^{\mu-1}+\mu\left|a_{n-\mu}\right|}\right] \mathrm{e}^{i \theta}\right|\right|^{\phi \delta} \mathrm{d} \theta\right\}^{\frac{1}{q \delta}}\left\{\int_{0}^{2 \pi}\left|P^{\prime}\left(\mathrm{e}^{i \theta}\right)\right|^{p \delta} \mathrm{d} \theta\right\}^{\frac{1}{p \delta}}
\end{aligned}
$$

which proves the desired result.

\section{Acknowledgements}

The authors are grateful to the referee for useful comments.

\section{Author details}

${ }^{1}$ Bharathiar University, Coimbatore, TN, 641046, India ${ }^{2}$ Department of Mathematics, Kashmir University Srinagar, 190006, India

\section{Authors' contributions}

GS studied the related literature under the supervision of WMS and jointly developed the idea and drafted the manuscript. GS made the text_le and communicated the manuscript. GS also revised it as per the directions of the referee under the guidance of WMS. Both the authors read and approved the final manuscript.

\section{Competing interests}

The authors declare that they have no competing interests. 


\section{References}

1. Turan, P: Über die Ableitung von Polynomen. Composito Math. 7, 89-95 (1939)

2. Malik, MA: On the derivative of a polynomial. J Lond Math Soc. 1(2), 57-60 (1969)

3. Malik, MA: An integral mean estimate for polynomials. Proc Am Math Soc. 91(2), 281-284 (1984). doi:10.1090/S0002 9939-1984-0740186-3

4. Aziz, A, Shah, WM: An integral mean estimate for polynomials. Indian J Pure Appl Math. 28(10), 1413-1419 (1997)

5. Aziz, A, Ahmad, N: Integral mean estimates for polynomials whose zeros are within a circle. Glas Mat Ser III. 31((51)2), 229-237 (1996)

6. Aziz, A, Dawood, QM: Inequalities for a polynomial and its derivative. J Approx Theory. 54, 306-313 (1988). doi:10.1016/ 0021-9045(88)90006-8

7. Qazi, MA: On the maximum modulus of polynomials. Proc Am Math Soc. 115, 337-343 (1990)

8. Govil, NK, Mc Tume, GN: Some generalizations involving the polar derivative for an inequality of Paul Turan. Acta Math Thunder. 104(1-2), 115-126 (2004)

9. Rahman, QI, Schmeisser, G: Analytic Theory of Polynomials. Oxford University Press, New York (2002)

10. Milovanovic, GV, Mitrinovic, DS, Rassias, ThM: Topics in Polynomials, Extremal Problems, Inequalities, Zeros. World Scientific, Singapore (1994)

11. Hille, E: Analytic Function Theory, Vol. II, Introduction to Higher Mathematics. Ginn and Company, New York, Toronto (1962)

doi:10.1186/1029-242X-2011-35

Cite this article as: Singh and Shah: Integral mean estimates for polynomials whose zeros are within a circle. Journal of Inequalities and Applications $20112011: 35$

\section{Submit your manuscript to a SpringerOpen ${ }^{\circ}$} journal and benefit from:

- Convenient online submission

- Rigorous peer review

- Immediate publication on acceptance

- Open access: articles freely available online

- High visibility within the field

- Retaining the copyright to your article

Submit your next manuscript at $\gg$ springeropen.com 